\title{
Effect of Polyelectrolyte Structure on Protein-Polyelectrolyte Coacervates: Coacervates of Bovine Serum Albumin with Poly(diallyldimethylammonium chloride) versus Chitosan
}

\author{
A. Basak Kayitmazer, ${ }^{*},+\perp$ Sabina P. Strand, ${ }^{\ddagger}$ Christophe Tribet,, Werner Jaeger," and \\ Paul L. Dubin*, $†$ \\ Department of Chemistry, University of Massachusetts-Amherst, 710 North Pleasant Street, Amherst, \\ Massachusetts 01003, NOBIPOL, Department of Biotechnology, The Norwegian University of Science and \\ Technology, NO-7491 Trondheim, Norway, Laboratoire de Physico-Chimie des Polymeres, \\ UMR CNRS 7615, ESPCI, Université Paris 6, 75231 Paris, France, and Fraunhofer-Institut \\ für Angewandte Polymerforschung, D-14476 Potsdam-Golm, Germany
}

Received June 9, 2007; Revised Manuscript Received July 28, 2007

\begin{abstract}
Electrostatic interactions between synthetic polyelectrolytes and proteins can lead to the formation of dense, macroion-rich liquid phases, with equilibrium microheterogeneities on length scales up to hundreds of nanometers. The effects of $\mathrm{pH}$ and ionic strength on the rheological and optical properties of these coacervates indicate microstructures sensitive to protein-polyelectrolyte interactions. We report here on the properties of coacervates obtained for bovine serum albumin (BSA) with the biopolyelectrolyte chitosan and find remarkable differences relative to coacervates obtained for BSA with poly(diallyldimethylammonium chloride) (PDADMAC). Coacervation with chitosan occurs more readily than with PDADMAC. Viscosities of coacervates obtained with chitosan are more than an order of magnitude larger and, unlike those with PDADMAC, show temperature and shear rate dependence. For the coacervates with chitosan, a fast relaxation time in dynamic light scattering, attributable to relatively unrestricted protein diffusion in both systems, is diminished in intensity by a factor of $3-4$, and the consequent dominance by slow modes is accompanied by a more heterogeneous array of slow apparent diffusivities. In place of a small-angle neutron scattering Guinier region in the vicinity of $0.004 \AA^{-1}$, a 10 -fold increase in scattering intensity is observed at lower $q$. Taken together, these results confirm the presence of dense domains on length scales of hundreds of nanometers to micrometers, which in coacervates prepared with chitosan are less solidlike, more interconnected, and occupy a larger volume fraction. The differences in properties are thus correlated with differences in mesophase structure.
\end{abstract}

\section{Introduction}

Chitosan, a linear cationic polysaccharide most commonly obtained by alkaline deacetylation of chitin, is a copolymer of $\beta$-(1 $\rightarrow 4$ )-2-amino-2-deoxy-D-glucopyranose and $\beta$-(1 $\rightarrow 4)$-2acetamino-2-deoxy-D-glucopyranose. Interest in chitosan has been increasing exponentially: The number of relevant papers has risen from 123 in 1991 to 1566 in $2006 .{ }^{1}$ In addition to being one of the few cationic biopolymers in nature, chitosan is biocompatible, biodegradable, nonimmunogenic, and nontoxic in animal tissues. While chitosan is already commercialized as a wound-healing agent, it also has been proposed as a disintegrant for tablets, a vehicle or coating material for controlled release or drug delivery, an absorption promoter for peptide and protein drugs, and a cell carrier for tissue repair. ${ }^{2-6}$ Nonbiomedical applications involve edible antifungal coating for the food industry, ${ }^{7}$ enzyme immobilization for bioreactors,, 89 fat trapping in the cosmetics industry, ${ }^{10}$ and low-cost removal of heavy metals from wastewater. ${ }^{11}$ Last, chitosan may serve

* Authors to whom correspondence should be addressed. E-mail: kayitmazer@northwestern.edu; dubin@chem.umass.edu.

$\dagger$ University of Massachusetts Amherst.

$\doteqdot$ The Norwegian University of Science and Technology.

$\S$ Université Paris 6.

"Fraunhofer-Institute für Angewandte Polymerforschung.

${ }^{\perp}$ Present address: Northwestern University, Biomedical Engineering, Evanston, IL 60208. as an interface between biological sensing molecules and microfabricated devices for biosensor design. ${ }^{6}$

Many of the preceding applications involve interactions between chitosan and peptides or proteins. The interaction of chitosan with proteins is mainly electrostatic, although hydrophobic forces may always be invoked, and chitosan aggregation and precipitation at high $\mathrm{pH}$ is a complicating factor. Takeuchi et al. ${ }^{12}$ studied chitosan-soybean $\beta$-conglycinin interactions by circular dichroism (CD) spectroscopy. The increase in the thermal unfolding temperature of the enzyme in the presence of chitosan was attributed to the stabilization of protein through electrostatic interaction with chitosan. ${ }^{12}$ At $\mathrm{pH}>\mathrm{pI}$, where protein and chitosan have opposite charges, complex formation of faba bean legumin ${ }^{13}$ with chitosan gave a small positive enthalpy change in contrast to the strongly exothermic interaction of chitosan with $\beta$-lactoglobulin. ${ }^{14}$ However, these variable observations do not preclude predominantly electrostatic interaction: Schaaf and co-workers ${ }^{15}$ showed that complexation of oppositely charge polyelectrolytes can be either exothermic or endothermic.

The stiffness of chitosan-bare persistence length $L_{\mathrm{p}}^{\circ}$ of $6 \mathrm{~nm}$, independent of the degree of acetylation ${ }^{16}$-would appear to make it useful in testing the expectation that reduced polyelectrolyte flexibility impedes binding to oppositely charged colloidal particles. ${ }^{17-19}$ In conjunction with poly(diallydimethylammonium chloride) (PDADMAC), chitosan affords a homologous pair with similar cationic charge spacing (6 ̊) but very different 
flexibilities $\left(2.5 \mathrm{~nm}\right.$ for PDADMAC $\left.{ }^{20}\right)$. For this reason, Smidsrød and co-workers ${ }^{21}$ carried out stiffness parameter calculations for chitosan versus PDADMAC, confirming the 2-fold higher flexibility of PDADMAC over chitosan. It should be pointed out however that $L_{\mathrm{p}}^{\circ}$ corresponds to the tendency of the chain to propagate in a given direction over dimensions on the order of $R_{g}$ and has limited significance for the behavior at length scales less than $L_{\mathrm{p}}^{\circ}$, i.e., the radius of small colloids. This can lead to a discrepancy between the expectation of weaker binding for "more stiff" polyelectrolytes from simulations and intuition, on the one hand, and the results of experiment, on the other. ${ }^{22}$

In the course of comparing the complexation of bovine serum albumin (BSA) with chitosan versus PDADMAC, ${ }^{22}$ we obtained the unexpected result of coacervation (liquid-liquid phase separation) with chitosan at a protein negative charge significantly smaller than that required for coacervation with PDADMAC. In other words, chitosan coacervated with BSA only slightly beyond conditions for complex formation. Because coacervation can be useful for encapsulation or enzyme immobilization, control of the range of coacervation could have application consequences. The properties of the two coacervates were remarkably different, the one with chitosan displaying much larger viscosity. Because the term "coacervate" often refers to the metastable liquid-liquid suspension, it is important to point out that all of the measurements in here and in our previous work refer to the discontinuous phases, isolated by centrifugation as optically clear, viscous fluids, which, in contrast to their precursor suspensions, are true equilibrium systems, stable for months. ${ }^{23,24}$ Because the unusual properties of protein-polyelectrolyte coacervates (e.g., the absence of aggregation at high protein concentration, unusually large protein diffusivities coupled with large bulk viscosity) appear to arise from mesophase heterogeneity, ${ }^{24}$ the striking difference between coacervates prepared with chitosan vs PDADMAC appeared to offer clues about structure-property relations in proteinpolyelectrolyte coacervates. We report here results from rheology, dynamic light scattering, and small-angle neutron scattering that support the proposal that the structure and dynamics of equilibrium mesophase dense domains dominate the behavior of these fluids.

\section{Experimental Section}

Materials. Poly(dimethyldiallyammonium chloride) (PDADMAC) of $M_{\mathrm{w}}=219 \mathrm{kDa}\left(M_{\mathrm{n}}=141 \mathrm{kDa}\right)$ and $M_{\mathrm{w}}=700 \mathrm{kDa}\left(M_{\mathrm{n}}=460\right.$ $\mathrm{kDa}$ ) samples were prepared by free radical aqueous polymerization of diallydimethylammonium chloride ${ }^{20}$ and characterized after dialysis and lyophilization by membrane osmometry and light scattering. Chitosan was prepared by homogeneous de-N-acetylation of shrimp chitin as previously described, ${ }^{25}$ converted to the $\mathrm{HCl}$ salt, ${ }^{26}$ and lyophilized. Characterization by osmometry, viscosity measurements, and ${ }^{1} \mathrm{H}$ NMR ${ }^{27}$ revealed the following properties, respectively: $M_{\mathrm{n}}=$ $150 \mathrm{kDa},[\eta]=600 \mathrm{~mL} / \mathrm{g}$, and degree of acetylation $<0.1 \%$. Because coacervation behavior appeared particularly sensitive to the last variable, it was considered important to minimize this source of heterogeneity. Bovine serum albumin (BSA) $\left(M_{\mathrm{w}} \approx 68 \mathrm{kDa}\right)$ with total fatty acid content $\leq 1.2 \mathrm{mg} / \mathrm{g}$ was purchased from Roche Diagnostics (Indianapolis, IN). Deuterium oxide (D, 99.8\%) was purchased from Cambridge Isotope Laboratories, Inc. (Andover, MA). $\mathrm{HCl}(0.1 \mathrm{~N})$ and $\mathrm{NaOH}(1$ N) solutions were obtained from Fisher Scientific. Milli-Q water was used in all sample preparations.

Methods. Coacervate Preparation. BSA (6 g/L) and polymer $(1.2$ $\mathrm{g} / \mathrm{L}$ ) were prepared separately in $0.05-0.1 \mathrm{M} \mathrm{NaCl}$ solution and filtered $\left(0.20 \mu \mathrm{m}\right.$ cellulose acetate, Sartorius, Inc.). PDADMAC $M_{\mathrm{w}}=219$

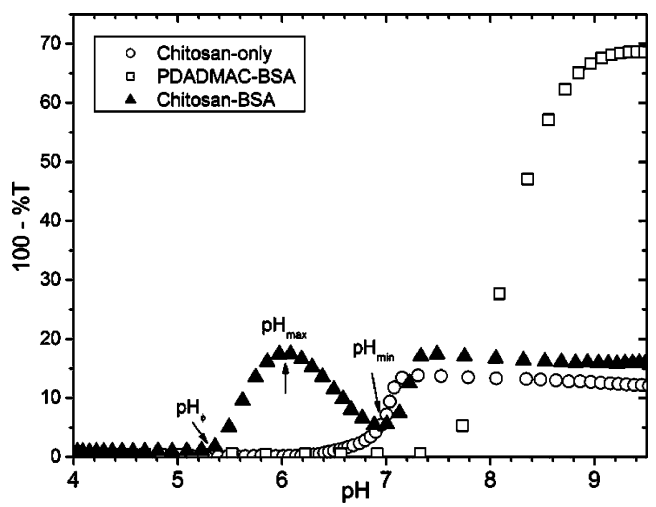

Figure 1. Turbidity vs $\mathrm{pH}$ for chitosan-BSA, PDADMAC-BSA, and chitosan alone in $I=100 \mathrm{mM} \mathrm{NaCl}$. $\mathrm{pH}_{\varphi}, \mathrm{pH}_{\max }(\mathrm{pH}$ of maximum turbidity for coacervation), and $\mathrm{pH}_{\min }(\mathrm{pH}$ of minimum turbidity for coacervate dissolution) correspond to the chitosan-BSA coacervation. (See text for explanation.)

$\mathrm{kDa}$ was used for most of the PDADMAC-BSA coacervates except for one prepared at $\mathrm{pH}=7.7$ and $I=0.1 \mathrm{M} \mathrm{NaCl}$, where the highermolecular-weight polymer was employed to increase the coacervate yield. To avoid unintended phase separation and to ensure the full solubility of chitosan, which diminished at $\mathrm{pH} \geq 7, \mathrm{NaCl}$ solutions were brought to $\mathrm{pH}<4.0$ before adding either polymer. The $\mathrm{pH}$ of each solution was adjusted to 4.0 (non-interacting conditions) with 0.1 and $1 \mathrm{~N} \mathrm{HCl}$ solution for polymer and protein, respectively. The two solutions were mixed $1: 1 \mathrm{v} / \mathrm{v}$ to give an initial protein/polymer weight ratio $(r)$ of 5 and adjusted to the desired $\mathrm{pH}$ by gradual addition with $\mathrm{NaOH}$, first $1 \mathrm{~N}$, then $0.1 \mathrm{~N}$. For coacervates with PDADMAC, this $\mathrm{pH}$ varied from 7.7 to 9.0 but for chitosan coacervation was essentially limited to 5.8 at 50 and $100 \mathrm{mM} \mathrm{NaCl}$ (Figure 1). Under these conditions the yield with chitosan was $1 \%$, while the yield with PDADMAC varied from $0.1 \%$ at $\mathrm{pH} 7.7,100 \mathrm{mM}$ salt, to $1 \%$ at $\mathrm{pH}$ $8.5, I=100 \mathrm{mM}$, or $\mathrm{pH} 9.0, I=50 \mathrm{mM}$. To obtain sufficient sample for small-angle neutron scattering (SANS) for PDADMAC at the lower $\mathrm{pH}$, we employed the higher-molecular-weight PDADMAC in that case. The mixture at room temperature was centrifuged for $30 \mathrm{~min}$ at 4000 $\mathrm{rpm}$, and the upper clear solution (supernatant) was removed by pipette from the lower optically clear and viscous fluid (coacervate). Centrifugation was repeated several times to ensure full removal of supernatant; any less stringent methods were likely to lead to nonequilibrium states for the two phases. For the chitosan-BSA coacervate, centrifugation was done at $22 \pm 3{ }^{\circ} \mathrm{C}$ to avoid additional temperature-induced phase separation within the coacervate phase at the lower temperatures. PDADMAC-BSA coacervates prepared in this manner are remarkably stable, as verified by the reproducibility of the dynamic light scattering (DLS) spectra after storage for 4 months, even when the three modes are resolved. ${ }^{23}$ These spectra were also found to be reproducible among samples prepared by different workers using slightly different procedures. Similar reproducibility was found for chitosan-BSA coacervates, attesting to the true equilibrium state of these complex fluids.

For SANS measurements, an $\mathrm{H}_{2} \mathrm{O} / \mathrm{D}_{2} \mathrm{O}$ exchange procedure employed a polymer-BSA mixture at a $\mathrm{pH}, I$, protein concentration, and polymer concentration identical to the appropriate supernatant but with $\mathrm{D}_{2} \mathrm{O}$ instead of $\mathrm{H}_{2} \mathrm{O}$. This solution was mixed with the corresponding coacervate, tumbled for 2-4 h, centrifuged, and removed from its supernatant. This process was repeated to reach an estimated content of $\mathrm{D}_{2} \mathrm{O}$ in the coacervate of $90 \%$.

Composition Determination. The water content of chitosan-BSA coacervates was determined by dry-weight analysis. Protein content was determined by UV-vis spectrometry. The content of chitosan was determined by reaction with ninhydrin. ${ }^{28}$ To remove interference from protein, the coacervate was incubated with concentrated nitric acid for $4 \mathrm{~h}$ at $70{ }^{\circ} \mathrm{C}$ followed by dilution of the resultant solution with water, dialysis (Piercenet, molecular weight cutoff of 3500), and filtration with $0.45 \mu \mathrm{m}$ cellulose acetate paper (Sartorius). 
Turbidimetric Titrations. Turbidity measurements, reported as 100 $-\% T$ were performed at ambient temperature using a Brinkman PC800 probe colorimeter at $420 \mathrm{~nm}$ equipped with a $2 \mathrm{~cm}$ path length fiberoptics probe. BSA $(0.6 \mathrm{~g} / \mathrm{L})$ and polymer (chitosan or PDADMAC) solutions $(0.12 \mathrm{~g} / \mathrm{L})$ for turbidimetric titrations were dissolved separately in salt solutions of $10,20,50$, and $100 \mathrm{mM} \mathrm{NaCl}$. The protein and salt solutions were filtered with $0.2 \mu \mathrm{m}$ cellulose acetate paper (Sartorius, Göttingen, Germany) followed by $\mathrm{pH}$ adjustment to less than 3.0. After mixing those two solutions in equal volumes, turbidity and $\mathrm{pH}$ were recorded following addition of $0.1 \mathrm{~N} \mathrm{NaOH}$ ("type 1 turbidimetric titration"). A Corning $240 \mathrm{pH}$ meter equipped with a Beckman electrode was used for $\mathrm{pH}$ measurements.

Small-Angle Neutron Scattering and Ultra-Small-Angle Scattering. SANS experiments were performed at the $30 \mathrm{~m} \mathrm{NG3}$ instrument at the National Institute of Standards and Technology (NIST), NIST Center for Neutron Research, in Gaithersburg, MD. ${ }^{29}$ PDADMAC-BSA and chitosan-BSA coacervates were loaded into demountable titanium cells with sample thicknesses of 2 and $1 \mathrm{~mm}$, respectively (the smaller cell being difficult to load with the higher-viscosity chitosan coacervates). Total scattering intensity was measured at sample-detector distance configurations of 1 and $4 \mathrm{~m}$ at a neutron wavelength $(\lambda)$ of $6 \AA$ and with a lens configuration at $8.4 \AA$. Run times were $10 \mathrm{~min}$ for the $1 \mathrm{~m}$ configuration, $15 \mathrm{~min}$ for the $4 \mathrm{~m}$ configuration, and 25-180 $\mathrm{min}$ for the lens configuration. Raw SANS data were subtracted from the empty cell and background $\left(90 \% \mathrm{D}_{2} \mathrm{O}\right.$ and $\left.10 \% \mathrm{H}_{2} \mathrm{O}\right)$ scattering to obtain the absolute intensity. ${ }^{30}$ Because coacervate samples scattered isotropically, the data were reduced to one-dimensional dependence, $I(q)$. The scattering wave vector $(q)$ range accessible by the combined data set of the three configurations was $0.001-0.4 \AA^{-1}$. All SANS fits were performed by the use of interactive procedures of Igor (Wavemetrics, Inc., Portland, OR) data analysis software. ${ }^{30}$

Ultra-small-angle scattering (USANS) experiments were performed at the Bonse-Hart-type instrument ${ }^{31}$ on beam port BT-5 at NIST. Coacervate samples were loaded into demountable titanium cells with $5 / 8$ in. Gd apertures. PDADMAC-BSA coacervates were loaded into cells with $7.6 \mathrm{~mm}$ sample thickness whereas chitosan-BSA coacervates required $4 \mathrm{~mm}$ sample thickness and an additional $0.5 \mathrm{in}$. Cd mask due the limited amount of sample.

Dynamic Light Scattering. DLS was carried out with a Brookhaven BI-9000AT goniometer and digital autocorrelator (Holtsville, NY) employing a $488 \mathrm{~nm} 100 \mathrm{~mW}$ argon-ion laser. Measurements were carried out at ambient temperature and at a $90^{\circ}$ scattering angle. Diffusion coefficients, $D$, were obtained from the autocorrelator function by the use of non-negatively constrained least-squares (NNLS) and CONTIN fitting procedures. Because NNLS gives more robust results, the data reported here are exclusively from NNLS. (More detailed discussion about the selection of the fitting procedure is given in ref 23.)

Rheology. Rheological measurements were performed on a straincontrolled rheometer (Rheometrics Fluids spectrometer II, Rheometrics, Inc., Piscataway, NJ) fitted with cone-plate geometry $(25 \mathrm{~mm}$ diameter, gap $0.043 \mathrm{~mm})$. Successive measurements were performed at varying strain $(4-50 \%)$ and at two different temperatures, i.e. $11-12$ and 25 ${ }^{\circ} \mathrm{C}$. The elastic modulus $G^{\prime}$ and loss modulus $G^{\prime \prime}$ were obtained by subjecting the samples to dynamic oscillatory tests during which a sinusoidal strain was applied and the resulting stress was recorded. Shear rate viscosities were obtained both by increasing the shear rate and then decreasing it. The difference between forward and backward shearing was within the uncertainty of measurements, and only forward sweeps are presented here.

Cryogenic Transmission Electron Microscopy. Cryogenic transmission electron microscopy (CryoTEM) was performed for a PDADMAC-BSA coacervate prepared at $\mathrm{pH}=8.5$ and $I=100 \mathrm{mM} \mathrm{NaCl}$ by the group of Y. Talmon, Technion, Haifa. Sample preparation, transfer, and imaging were done following a procedure described elsewhere ${ }^{32}$ with the addition of a Gatan 626 cryo-holder system. The

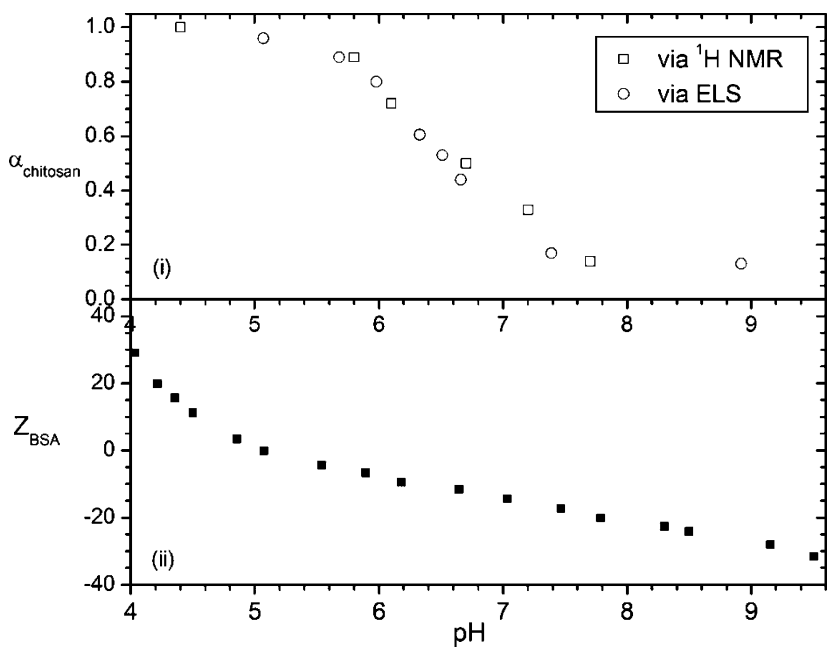

Figure 2. $\mathrm{pH}$ titration profiles of (i) chitosan ( $\alpha_{\text {chitosan, degree of }}$ protonation of chitosan) and (ii) BSA (net protein charge for BSA calculated from ref 35 and corrected for $\mathrm{Cl}^{-}$binding according to ref 36): ( $\square){ }^{1} \mathrm{H}$ NMR data; ${ }^{37}(\mathrm{O})$ electrophoretic light scattering data in panel i. ${ }^{27}$

images were taken with an FEI T12 G2 transmission electron microscope with a Gatan US1000 CCD camera.

\section{Results}

Turbidimetric Titrations. Figure 1 shows "type 1" titrations at $I=100 \mathrm{mM} \mathrm{NaCl}$ for BSA with either PDADMAC or chitosan and for chitosan alone. The region of soluble complex formation cannot be viewed in the scale of Figure 1 and is not directly relevant to the coacervate properties, the main subject here. Chitosan coacervates were prepared only at $\mathrm{pH} 5.8$, the condition for optimal yield close to $\mathrm{pH}_{\max }$ in Figure 1. The $\mathrm{pH}$ for the onset of coacervate formation, $\mathrm{pH}_{\phi},{ }^{23}$ is found to always be lower for chitosan than that for PDADMAC by $0.2-2 \mathrm{pH}$ units in $10-100 \mathrm{mM} \mathrm{NaCl}$, and the turbidity of the coacervate is substantially lower. The lower $\mathrm{pH}_{\phi}$ for the chitosan system could arise from a different mechanism of achieving complex charge neutrality, often considered a necessary condition for coacervation. ${ }^{33,34}$ For the chitosan system, this state can be approached by the reduction of chitosan positive charge with increasing $\mathrm{pH}$ (Figure 2), while for PDADMAC it can only be approached by the binding of negatively charged BSA; in other words binding becomes stronger with $\mathrm{pH}$ for the strong polycation PDADMAC, whereas for the chitosan system it could in fact weaken. A direct consequence is the redissolution of the chitosan coacervate at $\mathrm{pH} 6.8$; to verify that the measured turbidities between $\mathrm{pH}_{\max }$ and $\mathrm{pH}_{\min }$ are not time-dependent, we obtain identical curves when we reduced the rate of addition of $\mathrm{NaOH}$ by a factor of 4 . The coacervation of chitosan under such conditions of weak protein binding are likely to result in reduced counterion expulsion and desolvation; indeed, the water content is $84 \%$, in contrast to a typical value of $75 \%$ for PDADMAC-BSA coacervates, ${ }^{23}$ and the macromolecular concentrations are correspondingly lower, $14 \pm 2 \%$ BSA and $1.3 \pm 0.1 \%$ chitosan, compared to typical values of $20.5 \%$ BSA and $3.5 \%$ PDADMAC. Weaker binding might also arise from a reduction in polyelectrolyte flexibility; however, chain stiffness could favor coacervation, as one might expect that the loss of configurational entropy when the polymer is confined to the smaller coacervate volume will be less for a more rigid polymer. From the values above, we obtain protein/polymer ratios $r_{\text {coacervate }}$ of 11 and 6 for chitosan and PDADMAC, respectively. The 


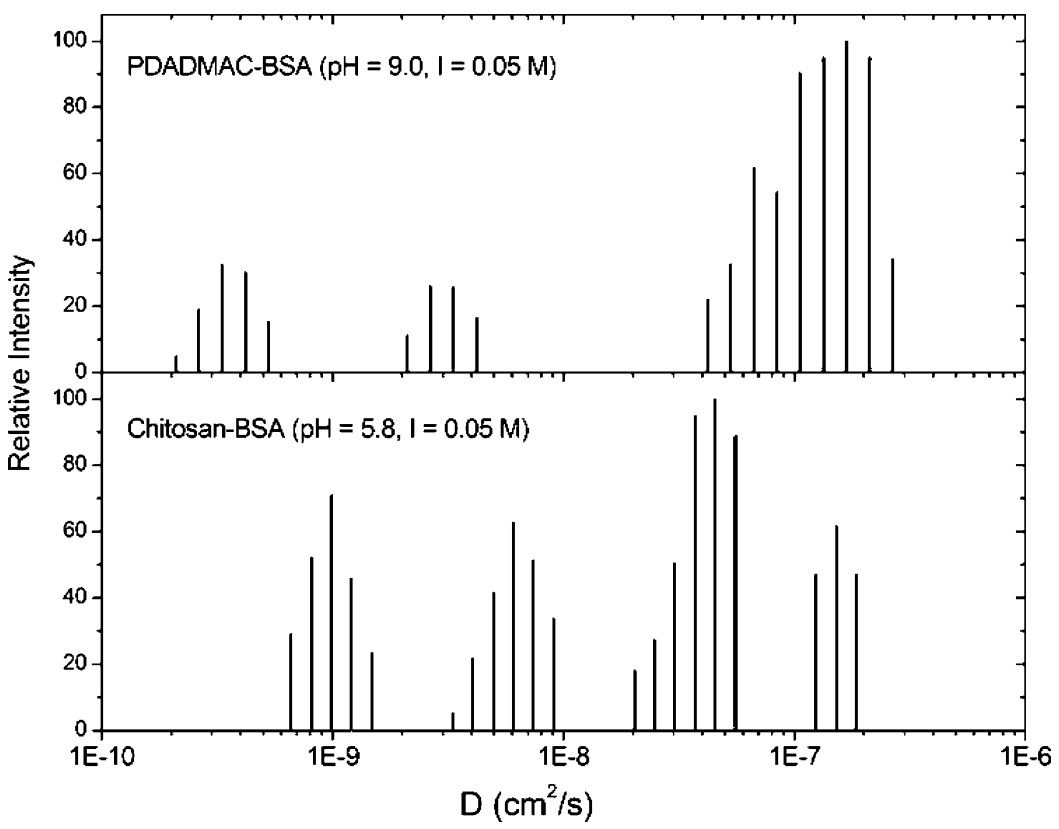

Figure 3. Relative intensity vs apparent $D$ for coacervates prepared with PDADMAC-BSA and chitosan-BSA. The values in parentheses are the conditions used to make the coacervate not the actual $\mathrm{pH}$ of the coacervate at the time of measurement.

large value of $r_{\text {coacervate }}$ for chitosan is consistent with the lower coacervation $\mathrm{pH}$, requiring more protein per polymer chain to achieve charge stoichiometry, but is lower by a factor of 4 than the number of proteins that might "fit" along the contour length. The contour length of PDADMAC (530 $\mathrm{nm}$ for $140 \mathrm{kDa})$ is very near that of chitosan $(560 \mathrm{~nm}$ for $150 \mathrm{kDa})$, but the number of proteins per polymer chain in that coacervate is smaller than the value for chitosan. This is as expected from the higher coacervation $\mathrm{pH}$ and shows the limited relevance of contour length. From UV analysis, we find BSA concentrations in the supernatants of $0.08 \%$ and $0.015 \%$, for chitosan and PDADMAC, respectively, and obtain rough estimates of $r_{\text {supernatant }}$ of 2 and 4 (compared to the initial stoichiometry of 5). Both coacervates contain at least $75 \%$ of the initial protein and about $20 \%$ of the initial polymer, although the value for the chitosan coacervate is rather unreliable due to the complexity of the method.

The plateau for the chitosan-BSA system in Figure 1 at $\mathrm{pH}$ $>7.2$ closely follows the plot for chitosan alone and is therefore attributable to chitosan precipitation due to the loss of charge (Figure 2). The decreased solubility of chitosan at $\mathrm{pH}>7$ also has been observed by others. ${ }^{38}$ An additional consequence of deprotonation of chitosan concurrent with the increase in protein charge is the dissolution of coacervates observed at $6.2<\mathrm{pH}$ $<7$. Changing the rate of base addition by a factor of 5 gave identical turbidimetric curves up to $\mathrm{pH} 8$, indicating that the maximum and minimum in Figure 1 do not arise from timedependent effects but reflect equilibrium states. The dissolution of coacervates could be due to the reduced binding of BSA or possible complex charge reversal at $\mathrm{pH}>6.2$, but zeta potential measurements failed to support the latter, suggesting instead the release of bound BSA. At pH 6.2, chitosan has lost about $35 \%$ of its positive charge (Figure 2), and the binding of BSA has presumably become quite weak.

Dynamic Light Scattering. DLS (Figure 3) reveals four modes of apparent diffusion coefficients for chitosan-BSA coacervates but only three for PDADMAC-BSA coacervates, the latter previously referred to as the fast and slow modes $\mathrm{F}$, $\mathrm{S} 1$, and S2. ${ }^{23}$ Given the potential fragility of the deconvolution of autocorrelation functions, we assured that the modes were
Table 1. Average Diffusion Coefficient for Coacervates in $I=50$ $\mathrm{mM} \mathrm{NaCl}$

\begin{tabular}{ccc}
\hline & \multicolumn{2}{c}{$D \times 10^{-7} \mathrm{~cm}^{2} / \mathrm{s}$ (relative amplitude \%) } \\
\cline { 2 - 3 } & $\begin{array}{c}\text { chitosan-BSA } \\
\mathrm{pH}=5.8, I=50 \mathrm{mM})\end{array}$ & $\begin{array}{c}\text { PDADMAC-BSA } \\
(\mathrm{pH}=9.0, I=50 \mathrm{mM})\end{array}$ \\
\hline $\mathrm{F} 1$ & $1-2(16)$ & $1.2(76)$ \\
$\mathrm{F} 2$ & $0.4(39)$ & - \\
S1 & $0.06(22)$ & $0.03(5)$ \\
S2 & $0.01(23)$ & $0.004(19)$ \\
\hline
\end{tabular}

highly reproducible and robust (more so for NNLS than for CONTIN (not shown)). For chitosan-BSA coacervates, two fast modes are identified: F1 (identical to the fast mode typically seen for PDADMAC coacervates) and a second fast mode F2. In the PDADMAC-BSA coacervates, F1 has been attributed to the "free" BSA diffusion in the dilute domains, ${ }^{23,24}$ but in this case it is not the dominant mode. F2 appears only in the chitosan-BSA coacervate and where it is the dominant mode (Table 1). The normalized intensity for F1 in the PDADMACBSA coacervates is larger than the total normalized intensity from F1 and F2 in the chitosan coacervates. This suggests that less protein is found in dilute domains for coacervates with chitosan, despite their larger water content.

Small-Angle Neutron Scattering. Neutron scattering spectra are shown in Figures 4 and 5 for the PDADMAC-BSA coacervates, prepared at $\mathrm{pH} 7.7,8.5$, and 9.0, and the chitosanBSA coacervates, prepared at $\mathrm{pH} 5.8$ and 5.9, at either $100 \mathrm{mM}$ or $50 \mathrm{mM}$ salt. Because the low $\mathrm{pH}$ coacervate with PDADMAC was prepared with high-molecular-weight polymer $\left(M_{\mathrm{n}}=460\right.$ $\mathrm{kDa}$ vs $140 \mathrm{kDa}$ for others) the difference in their two PDADMAC curves in Figure 4 might be related to either $\mathrm{pH}$ or molecular weight. Previous DLS and rheology data show no effect of molecular weight on coacervate structure in regard to DLS modes or modulus ${ }^{31}$ but strong effects of $\mathrm{pH}$ and ionic strength, so the influence of molecular weight in Figure 4 is secondary to the effect of $\mathrm{pH}$, and these two SANS curves will be considered below in terms of the different interaction strengths and not molecular weights. The most striking difference in the SANS profiles between the two systems is a 10fold increase in scattering intensity for the chitosan coacervate 


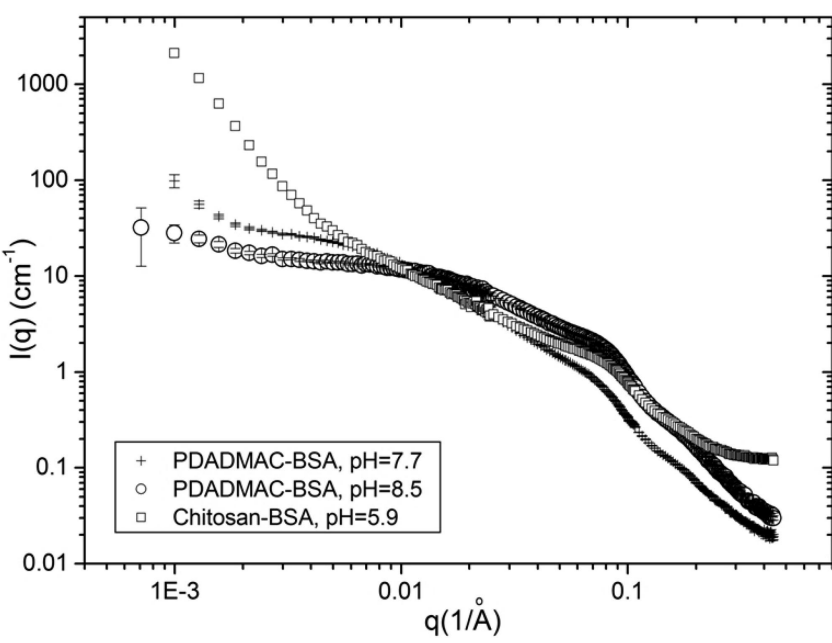

Figure 4. Scattering intensity distribution for the chitosan-BSA and PDADMAC-BSA coacervates prepared at $I=100 \mathrm{mM} \mathrm{NaCl}$ with $M_{\mathrm{n}}=140 \mathrm{kDa}$ at $\mathrm{pH} 8.5$ and $460 \mathrm{kDa}$ at $\mathrm{pH}$ 7.7. (The $\mathrm{pH}$ values given in the legend are preparation values of the coacervates.)

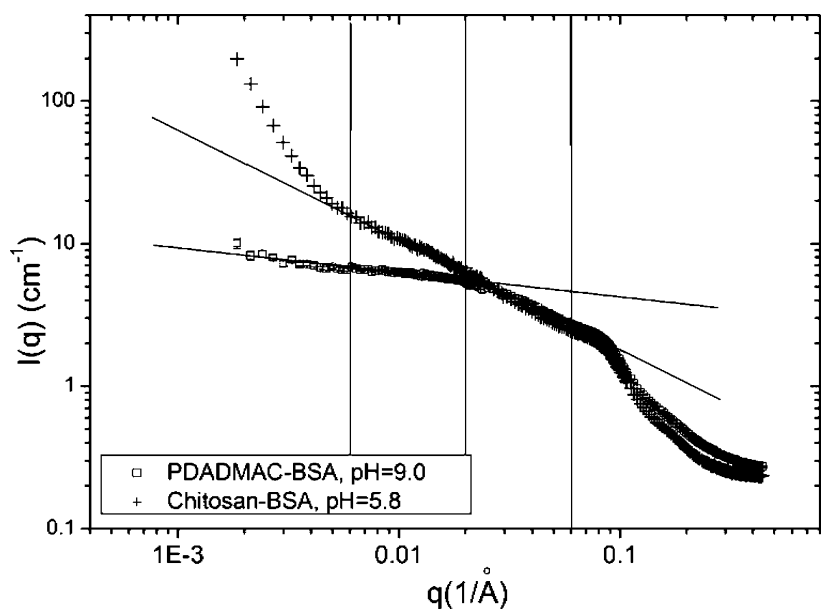

Figure 5. Scattering intensity profiles for the chitosan-BSA and PDADMAC-BSA coacervates prepared at $I=50 \mathrm{mM} \mathrm{NaCl}$. (The $\mathrm{pH}$ values given in the legend are preparation values of the coacervates.) Vertical lines are used to indicate $q$ ranges identified in the text as "low q", "first intermediate", and "second intermediate" regions (from left to right).

at $q<0.006 \AA^{-1}$, corresponding to length scales greater than $100 \mathrm{~nm}$. This upturn at low $q$ for coacervates with chitosan scales with $q^{-\alpha}$ where $\alpha$ is $2.8 \pm 0.1$, independent of ionic strength. This power-law exponent resembles the behavior of a three-dimensional object $(\alpha=3)$. The small upturns for the PDADMAC coacervates are likely to arise from smearing, as USANS profiles for these coacervates in the range $0.0002<q$ $<0.001$ (not shown) are flat and featureless, while the upturn is clearly seen with USANS for chitosan coacervates, although at a lower value of $\alpha=2.2$.

Scattering profiles are presented in Figure 5 for chitosan and PDADMAC prepared in $50 \mathrm{mM}$ salt to more clearly focus on differences between the two coacervates at a fixed ionic strength. In the first intermediate $q$ region $0.006<q<0.02 \AA^{-1}$, the presence of structure in the corresponding size range of $100-$ $30 \mathrm{~nm}$ is clearly more evident for chitosan. The slope of -0.9 for chitosan, compared to the almost negligible slope for the PDADMAC coacervates, is consistent with a one-dimensional structure for the former.

In the second intermediate region, $0.02<q<0.06 \AA^{-1}$, the profiles for the two systems are superimposible (Figure 5) and terminate in a peak that can be identified with the protein

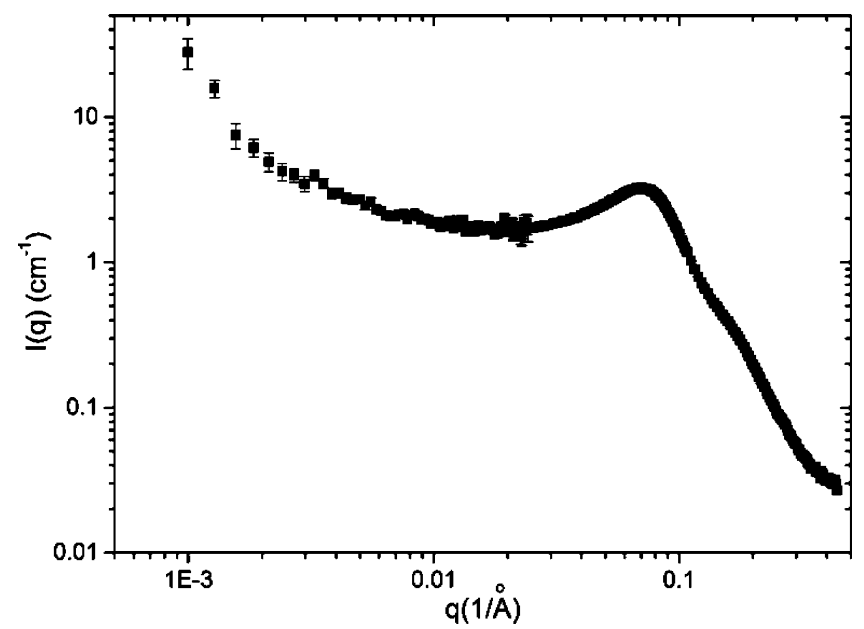

Figure 6. SANS intensity distribution for concentrated BSA solution at $\mathrm{pH} \approx 7$ in $100 \mathrm{mM} \mathrm{NaCl}$.

Table 2. Correlation Length for the Structure Peak in Coacervates and Concentrated BSA

\begin{tabular}{lcrrc}
\hline \multicolumn{1}{c}{ sample } & $\mathrm{pH}$ & \multicolumn{1}{c}{$\begin{array}{l}(\mathrm{M}) \\
\text { 230 g/L BSA }\end{array}$} & $\begin{array}{c}q^{*} \\
\left(\mathrm{~A}^{-1}\right)\end{array}$ & $\begin{array}{c}\text { correlation length } \\
(\mathrm{nm})\end{array}$ \\
\hline PDADMAC-BSA & 7.7 & 100 & 0.071 & 8.9 \\
PDADMAC-BSA & 8.5 & 100 & 0.076 & 8.3 \\
PDADMAC-BSA & 9.0 & 50 & 0.082 & 7.7 \\
chitosan-BSA & 5.8 & 50 & 0.084 & 7.5 \\
chitosan-BSA & 5.9 & 100 & 0.075 & 8.1 \\
\hline
\end{tabular}

correlation peak, as it appears most prominently in the profile of BSA alone (Figure 6) and is similar to ones seen by SANS for pectin- $\beta$-lactoglobulin coacervates ${ }^{39}$ concentrated lysozyme and cytochrome $\mathrm{C}$ solutions, ${ }^{40,41}$ and for $100 \mathrm{~g} / \mathrm{L} \mathrm{BSA}$ by smallangle X-ray scattering. ${ }^{42}$ While BSA is not a strongly aggregating protein, any aggregation would be greatest near its pI; however, as seen in Table 2, the protein peak correlation length is $7.8 \mathrm{~nm}$ at $\mathrm{pH} 5.9$ and $8 \pm 0.3 \mathrm{~nm}$ at $\mathrm{pH} 7.7-8.5(100 \mathrm{mM})$. On the other hand, the correlation length for BSA alone, 8.9 $\mathrm{nm}$, is significantly larger than the range in the coacervates. This diminution of the protein correlation length (Table 2) and the broadening of the peak relative to polymer-free BSA is an indication of a reduction in structure arising from diminished interprotein repulsion in the coacervates, the effect stronger at higher $\mathrm{pH}$ or lower $I$, where protein-polyelectrolyte interactions screen out protein-protein repulsions.

Examining this same second intermediate $q$ region more closely for the PDADMAC coacervates, we observe in Table 3 a monotonic decrease in the power-law dependence with increasing interaction strength (increasing negative protein charge). Because all of these terms are close to unity, they are consistent with one-dimensional structures, but the results of $\alpha$ $<1$ at low ionic strength, for both chitosan and PDADMAC, may indicate broken one-dimensional structures ${ }^{43,44}$ under conditions of stronger interaction.

Rheology. Shear-dependent viscosities are shown in Figure 7 for the chitosan coacervates at 12 and $25{ }^{\circ} \mathrm{C}$ and for the PDADMAC coacervates at $25{ }^{\circ} \mathrm{C}$. At $25{ }^{\circ} \mathrm{C}$ the viscosity at low shear $\eta_{0}$ is an order of magnitude larger for chitosan coacervates. $\eta_{0}$ for chitosan decreases about 5-fold with temperature increase; in comparison, the effect of temperature $(T)$ on $\eta_{0}$ of PDADMAC in a similar range of $T$ is only a factor of $2 .{ }^{23}$ The large temperature effect for the chitosan coacervates is much greater than the influence of temperature on chitosan 
Table 3. Net Protein Charge and Power-Law Dependence for Coacervates

\begin{tabular}{lcccccc}
\hline \multicolumn{1}{c}{ sample } & $\mathrm{pH}$ & $\begin{array}{c}l \\
(\mathrm{mM})\end{array}$ & $Z_{\mathrm{pr}}$ & $\begin{array}{c}q \text { range } \\
\left(\AA^{-1}\right)\end{array}$ & $\begin{array}{c}\text { corresponding size } \\
(\mathrm{nm})\end{array}$ & $\begin{array}{c}\text { power-law dependence } \\
(\alpha)\end{array}$ \\
\hline PDADMAC-BSA & 7.7 & 100 & -20 & $0.009-0.058$ & $70-11$ & 1.4 \\
PDADMAC-BSA & 8.5 & 100 & -24 & $0.020-0.060$ & $31-10$ & 1.04 \\
PDADMAC-BSA & 9.0 & 50 & -27 & $0.026-0.065$ & $24-10$ & 0.73 \\
chitosan-BSA & 5.8 & 50 & -6.5 & $0.0075-0.057$ & $84-11$ & 0.86 \\
chitosan-BSA & 5.9 & 100 & -6.6 & $0.006-0.045$ & $105-14$ & 1.1 \\
\hline
\end{tabular}

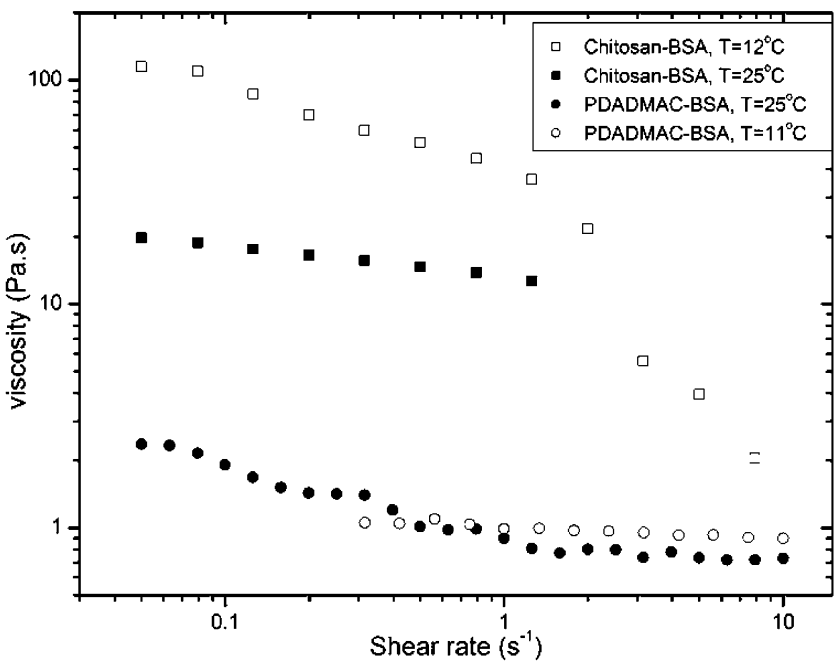

Figure 7. Shear rate viscosity of the PDADMAC-BSA and chitosanBSA coacervates at $T=12$ and $25^{\circ} \mathrm{C}$. The shear rate was increased progressively during the measurements. The data for the PDADMAC-BSA coacervate at $11^{\circ} \mathrm{C}$ are collected by dynamic viscosity measurements at $4 \%$ strain. Thus, the $x$-axis for those data corresponds to frequency $(\mathrm{rad} / \mathrm{s})$ rather than shear rate.

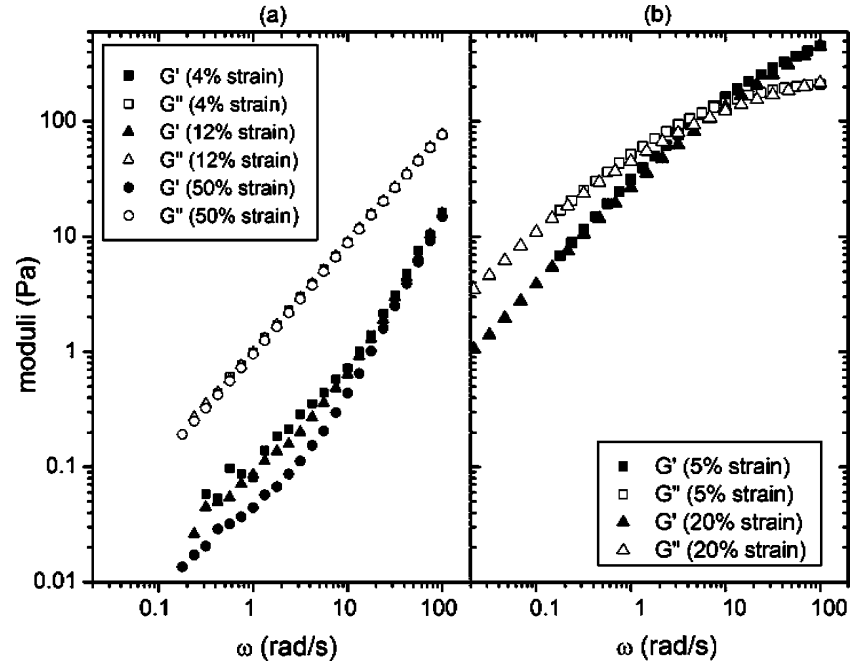

Figure 8. Effect of strain on frequency-dependent moduli for (a) the PDADMAC-BSA coacervate at $11^{\circ} \mathrm{C}$ (prepared at $\mathrm{pH}=9.0, I=50$ $\mathrm{mM} \mathrm{NaCl}$ ) and (b) the chitosan-BSA coacervate at $12{ }^{\circ} \mathrm{C}$ (prepared at $\mathrm{pH}=5.8, I=50 \mathrm{mM} \mathrm{NaCl}$ ).

alone: A $50 \mathrm{~g} / \mathrm{L}$ chitosan solution shows a decrease in $\eta$ of only $33 \%$ for the same temperature change. ${ }^{45}$

At $11{ }^{\circ} \mathrm{C}$, shear thinning occurs dramatically for chitosan coacervates, viscosity dropping 20-fold beyond a frequency of about $1 \mathrm{rad} / \mathrm{s}$, obviously in contrast to the PDADMAC coacervates at this temperature. A more continuous shear effect is seen for PDADMAC at $25^{\circ} \mathrm{C}$ but with only a 3-fold change in viscosity over the whole shear rate range. This shear-thinning behavior might reflect the much slower processes of strain-

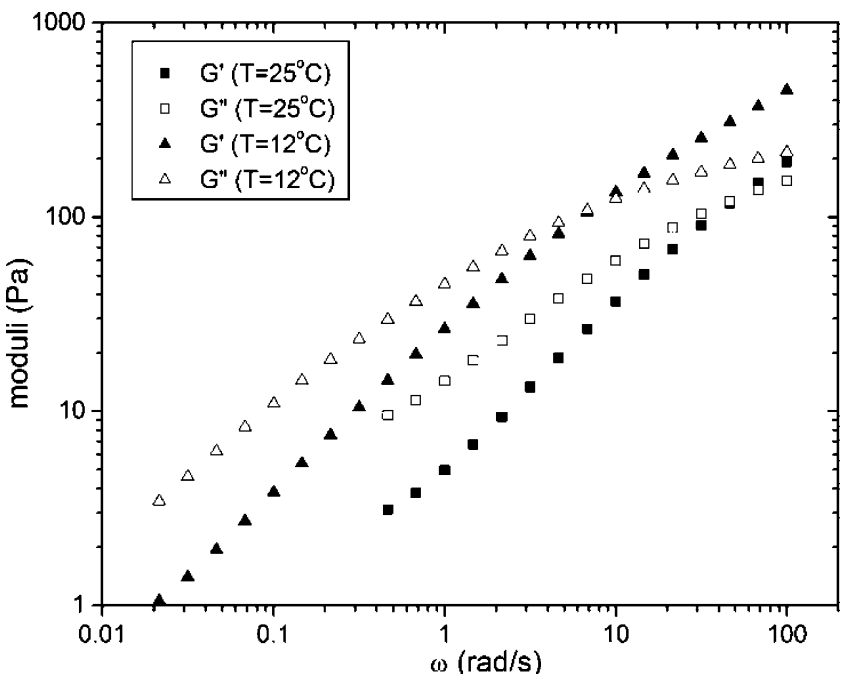

Figure 9. Effect of $T$ on dynamic moduli of the chitosan-BSA coacervate (prepared at $\mathrm{pH}=5.8, I=50 \mathrm{mM} \mathrm{NaCl}$ ) at a strain amplitude of $20 \%$.

relaxation in the chitosan coacervates at low temperatures, as further discussed below.

Frequency-dependent moduli for both coacervates at low temperatures are shown in Figure 8 for different strain amplitudes. There is no effect of strain up to $20 \%$ on $G^{\prime}$ or $G^{\prime \prime}$ for chitosan. For PDADMAC, the effect of strain can be said to disappear at $12 \%$ within the limits of experimental uncertainty. In contrast to the PDADMAC coacervates, which exhibit dominant viscous behavior at all frequencies $\omega$, consistent with the findings in ref 23 , chitosan coacervates show crossing of $G^{\prime}$ and $G^{\prime \prime}$ at $\omega \approx 10 \mathrm{~s}^{-1}$. Using the $G^{\prime}-G^{\prime \prime}$ crossover frequency at $\omega_{\times}$gives a characteristic relaxation time $\left(\tau=\omega_{\times}{ }^{-1}\right)$ of the visco-elastic network in the samples. The remarkable value of $\tau=150 \mathrm{~ms}$ in the chitosan coacervate, compared to the range of $0.5-2.5 \mathrm{~ms}$ in the PDADMAC samples, ${ }^{23}$ indicates an enormous increase in the lifetime of interchain connectivities for coacervates with chitosan. However, the elastic modulus at the crossover frequency of both the PDADMAC and the chitosan coacervates are on the order of $200 \mathrm{~Pa}$. Assuming that the elasticity arises from the network of interconnected chains, the similar elastic moduli suggests that the density of interchain contacts is similar in both samples.

The effect of temperature on the moduli for chitosan coacervates shown in Figure 9 is in general agreement with the depression of viscosity at the higher temperature of Figure 7. The decrease in both $G^{\prime}$ and $G^{\prime \prime}$ with increasing temperature is essentially due to a horizontal shift (frequency shift) of the frequency-moduli curves, and accordingly the modulus at the crossover between $G^{\prime}$ and $G^{\prime \prime}$ is not modified by temperature. The effect of temperature can be attributed primarily to a decrease in $\tau$ (from $150 \mathrm{~ms}$ at $12^{\circ} \mathrm{C}$ to $22 \mathrm{~ms}$ at $25^{\circ} \mathrm{C}$ ). While it has been proposed that the breaking of hydrogen bonds with temperature can account for the $6 \%$ increase in viscosity at the same temperature window for dilute chitosan, ${ }^{21}$ this effect is 
small compared to the changes seen in Figure 9. Altogether, the measurements indicate that the very large lifetimes of interchain connectivities for chitosan coacervates are highly susceptible to diminution by an increase in temperature.

\section{Discussion}

Although chitosan at $\mathrm{pH} 5.8$ has about the same linear charge density as PDADMAC, its coacervation with BSA and the properties of those coacervates are remarkably different. The outstanding features for those coacervates are: (1) formation at a $\mathrm{pH}$ only slightly above the $\mathrm{pI} ;$ (2) temperature- and sheardependent viscosity higher by 10-50 times; (3) appearance in DLS of an additional slow mode, together with strong reduction of the relative intensity of the fast mode, and a more heterogeneous array of slow apparent diffusivities; and (4) the greatly enhanced neutron scattering profile at $q<0.004 \AA$. We seek a model capable of explaining these differences, recognizing at the same time that a full understanding of structure-property relationships in the more thoroughly studied PDADMAC-BSA coacervates is still emerging.

Polyelectrolyte-colloid coacervation, as established by studies for PDADMAC and BSA and for PDADMAC and anionic/ nonionic mixed micelles, ${ }^{46,47}$ can be said to arise from extended interactions among soluble "primary" (e.g., intrapolymer) complexes, in a manner similar to the mechanism established for polyelectrolyte-polyelectrolyte coacervation by Veis. ${ }^{48}$ These interactions are inhibited for soluble complexes with a net charge, which to a first approximation approaches neutrality when the polyelectrolyte charge is compensated for by the opposing charge $n Z$ of bound colloids, where $Z$ is the charge per colloid and $n$ is the number of colloids bound per polyelectrolyte chain. This relation for total charge is approximate because selective retention and/or repulsion of counterions may contribute to charge balance. For cationic polyelectrolytes, charge reversal is observed as the colloid charge becomes more negative, ${ }^{49}$ because both $Z$ and $n$ increase in magnitude. The enhanced colloid-PE binding affinity due to increased nonspecific electrostatic interaction, can be accompanied by an increase in charge complementarity, and this "ion-pairing" leads to the entropically favorable expulsion of counterions and concomitant desolvation, an additional feature of coacervation. When charge complementarity or binding affinity is sufficiently large, counterion expulsion and desolvation may increase to the point that precipitation is seen instead of coacervation, as in salt-free solutions of proteins and oppositely charged polyelectrolytes. ${ }^{50}$ Charge neutralization and counterion expulsion may often coincide, but the former can occur without the latter.

In the case of PDADMAC-BSA, charge neutrality is achieved by an increase in $\left|z_{\mathrm{BSA}}\right|$ and $n$ with $\mathrm{pH},{ }^{49}$ but for chitosan, an increase in $\left|z_{\mathrm{BSA}}\right|$ (negative above $\mathrm{pH}=\mathrm{pI}=5$ ) is accompanied by a decrease in $\left|z_{\text {chitin }}\right|$ for $\mathrm{pH}>5.8$ (Figure 2), reflected in the redissolution of the coacervates at $6<\mathrm{pH}<$ 6.8 in $I=100 \mathrm{mM} \mathrm{NaCl}$ as shown in Figure 1. Thus, charge neutralization of soluble complexes can be approached at smaller $\left|z_{\mathrm{BSA}}\right|$, leading to coacervation at lower $\mathrm{pH}$. The more facile coacervation with chitosan could also arise from the decrease in the loss of chain configurational entropy for this more rigid polycation, although for polyelectrolyte-polyelectrolyte coacervation it has been proposed that chain configurational entropy might increase upon coacervation. ${ }^{48}$

In contrast to chitosan-BSA coacervates, the preparation of PDADMAC-BSA coacervates involves the addition of $\mathrm{NaOH}$ to coacervates initially formed at $\mathrm{pH}_{\phi}$ to attain $\mathrm{pH}$ 's $1-2.5 \mathrm{pH}$ units higher than $\mathrm{pH}_{\phi}$. Upon equilibration of suspended coacervate droplets with the basic continuous dilute phase, droplets become less transparent and eventually solidlike, ${ }^{51}$ evidence of desolvation due to more efficient ion-pairing (e.g., the replacement of $\mathrm{Na}^{+}$near protein negative domains by polycation chains), leading to the expulsion of microions. The reduction in water content from $85 \%$ to $74 \%$ for coacervates prepared at weak interaction conditions $(\mathrm{pH}=7.6, I=1 \mathrm{mM})$ versus stronger conditions $(\mathrm{pH}=9.5, I=100 \mathrm{mM})^{52}$ is additional evidence for the same effect. Centrifugation in our coacervate preparation fuses droplets containing complexes or aggregates thereof already somewhat desolvated. In coacervates, these aggregates must be elemental components of the mesophase dense domains previously deduced from DLS, rheology, and fluorescence recovery after photobleaching. ${ }^{23,24}$ It is not known whether a different method of coacervate preparation (e.g., mixing of protein and polyelectrolyte solutions preadjusted to $\mathrm{pH}>\mathrm{pH}_{\phi}$ ) would yield the same microstructure, but the repeatability of DLS spectra after months of storage and the reproducibility of data for samples prepared in different laboratories indicate the equilibrated nature of coacervates prepared by the present approach.

Chitosan-BSA coacervates must represent weaker proteinpolyelectrolyte electrostatic interactions than those in the PDADMAC-BSA coacervates: At the respective $\mathrm{pH}$ 's used in coacervate preparation, the protein negative charge is smaller by a factor of 3-4 for the former, and the polyelectrolyte positive linear charge density is also reduced (Figure 2). One manifestation of this weaker interaction is the reduced expulsion of water, lower by almost 2-fold for chitosan coacervates. The stiffness of the chitosan chain may also reduce charge complementarity. Thus, relative to chitosan at $\mathrm{pH} 5.8$, coacervation with PDADMAC at $\mathrm{pH}>7.5$ produces mesodomains with tighter ion-pairing and more desolvation. The resultant dense domains occupy relatively low volumes and tend to be disconnected. Conversely, the dense domains in coacervates prepared with chitosan are more voluminous and hence more interconnected. The corresponding reduced contrast of dilute domains in the chitosan-BSA coacervates explains the 2-fold reduction in relative scattering of the fast DLS mode. The greater interconnectedness of those domains explains their stronger SANS scattering seen at low $q(2 \pi / q>150 \mathrm{~nm})$, while the disconnected nature of dense domains for the PDADMACBSA coacervates explains the low power-law exponent in the $0.01<q<0.06 \AA^{-1}$ region.

An instantaneous snapshot of an intrapolymer soluble complex would reveal distal regions of lower macroion charge compensation that are electrostatically less favorable than interior regions. One driving force for higher-order aggregation of intrapolymer complexes ${ }^{51}$ is the elimination of these distal regions. The energy difference between soluble complexes and aggregates thereof can appear as an interfacial energy, promoting soluble complex fusion, which from a mechanistic point of view could be facilitated by charge polarizability. Boundaries at the surfaces of dense domains should be similarly destabilized, leading to their unlimited fusion in the coacervate, so we consider possible scenarios to account for the finite size of dense domains.

It has been proposed that a distribution of dense domains of different sizes could be stabilized relative to an infinite one by entropy. ${ }^{53,54}$ More specific to the current system, we can propose that at a high protein charge intrapolymer BSA-PDADMAC complexes (were they to exist discretely in the coacervate) could 


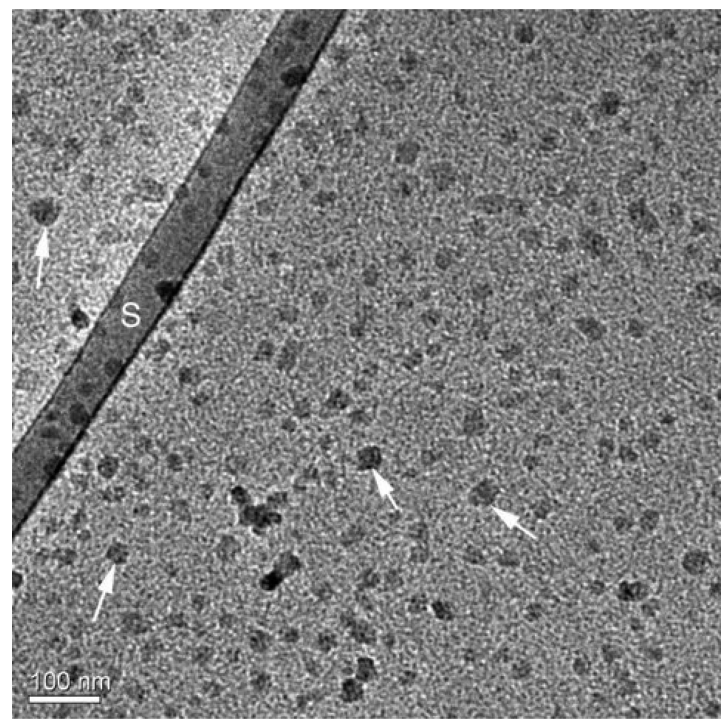

Figure 10. Cryo-TEM image of the PDADMAC-BSA coacervate prepared at $\mathrm{pH}=8.5, I=100 \mathrm{mM} \mathrm{NaCl}$. "S" denotes the support film. The scale bar is $100 \mathrm{~nm}$.

bear a net charge but still interact favorably with others via polarizability and the enhancement of polyelectrolyte-protein interactions when they fuse. This combination of short-range attraction with long-range repulsion due to charge accumulation has been discussed for the clustering of weakly charged colloidal silver ${ }^{54}$ and Boehmide rods ${ }^{53}$ as a mechanism for limited cluster growth. In the present case, the objects that form these clusters are likely to be aggregates: multipolymer species that represent the fusion of primary (intrapolymer) species that can undergo association up to certain degree of charge accumulation. Cluster formation is likely to proceed isotropically for highly charged aggregates, thus leading to more extended geometries (and low power-law exponents) in the case of the PDADMAC-BSA coacervates, particularly those formed at high pH (Table 3). Multipolymer complexes can be observed in BSA-PDADMAC solution, prior to phase separation, with hydrodynamic radii ranging from 25 to $150 \mathrm{~nm},{ }^{49,55}$ but the analogous species in coacervates should be desolvated, so a reasonable estimate for their diameters in coacervates would be on the order of 50$100 \mathrm{~nm}$. The smallest discernible discrete objects in a previously published CryoTEM image (Figure 4 in ref 24) appeared to have such dimensions, and these objects (aggregates) did appear to assemble into irregular clusters of hundreds of aggregates, although smaller clusters were observed as well. Such objects are also seen at higher resolutions in CryoTEM (Figure 10), although the nature of their interconnections appears more tenuous.

We now consider the differences between the chitosan-BSA and the PDADMAC-BSA coacervates, assuming the presence of clusters of charged aggregates, whose growth is limited by charge accumulation, at least for the latter. Relatively lower charges for both of the macroionic components of the chitosanBSA coacervates should reduce such charge accumulation, allowing for larger anisotropic cluster growth but with less desolvation. This could explain why dense domain scattering in SANS for the chitosan-BSA coacervates can be simultaneously diminished in intensity but also correspond to larger dimensions. The geometry of clusters could also be affected, yielding more extended (isotropic) but also disconnected dense domains for the more highly charged PDADMAC-BSA system, as schematically depicted in Figure 11A, leading to its lower power-law exponent (Figures 4 and 5). This hypothesis is
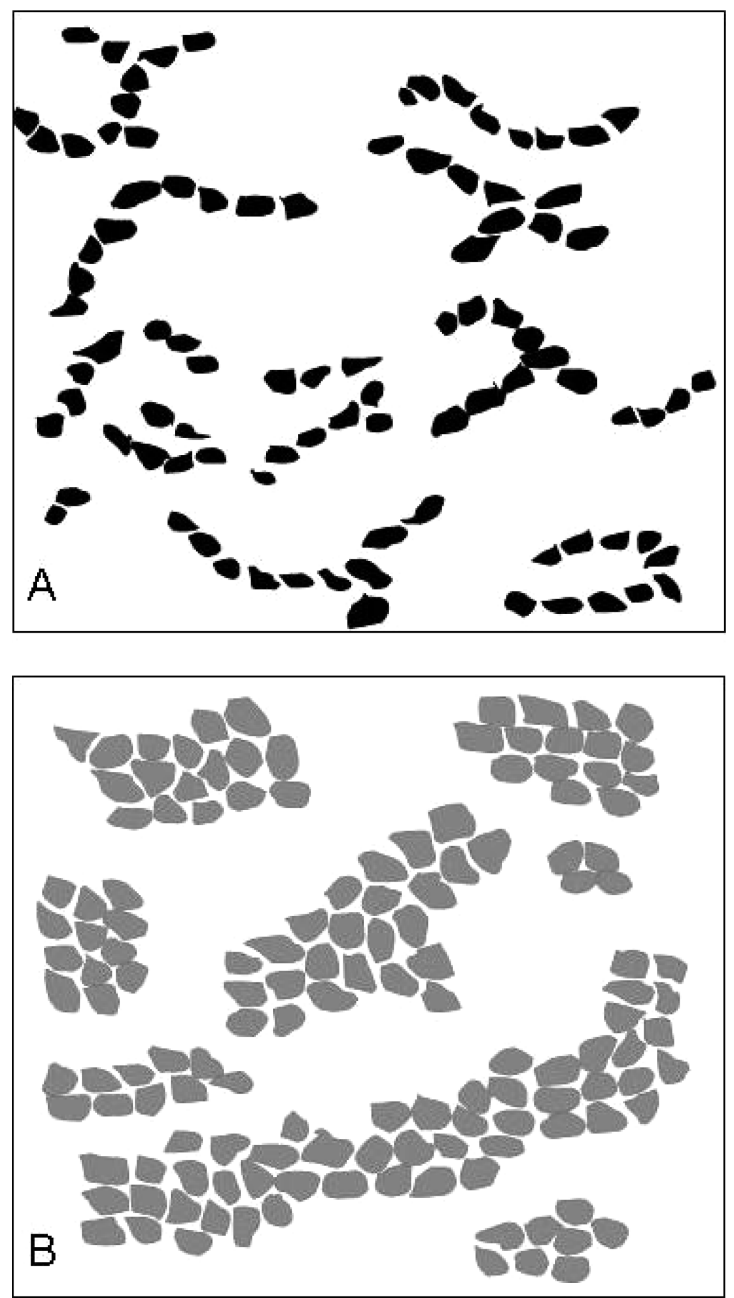

Figure 11. Models of coacervate structure for (A) PDADMAC-BSA and (B) chitosan-BSA. Partially desolvated multipolymer-protein complexes ("aggregates"), on the order of $50 \mathrm{~nm}$, form polydisperse isotropic clusters $(100-1000 \mathrm{~nm})$.

supported by a further decrease in that exponent with increasing protein charge (Table 3 ). Reduced connectivity at higher protein charge could also explain why the fast DLS mode for PDADMAC-BSA increases monotonically with $\mathrm{pH},{ }^{23,24}$ an effect previously overlooked, which we can now attribute to less constrained dilute mesophase volumes when clusters shrink. The dynamic breakup of these clusters is slowed down, leading to more prominent DLS slow modes for PDADMAC-BSA coacervates at high $\mathrm{pH}$ and low $I .^{23}$ Because connectivity between solid clusters would confer a dominant elastic character and obvious sensitivity to preshearing, ${ }^{23}$ the viscoelastic properties of the chitosan coacervates point to the loss of intercluster connectivity at large scales.

The proposed model is consistent in several ways with the pictures put forward by Gummel et al. ${ }^{56}$ based on SANS of turbid suspensions formed by mixing low low-molecular-weight sodium poly(styrene sulfonate) (PSS) with lysozyme in $50 \mathrm{mM}$ salt at $\mathrm{pH}$ 4.7. Gummel et al. identify "primary complexes" 15$30 \mathrm{~nm}$ in diameter, i.e., with sizes 8-15 times larger than the protein, which corresponds to $40-60 \mathrm{~nm}$ in our case. With $60-$ 500 proteins per primary complex, these strongly collapsed objects are analogous to our "aggregates". These relatively small and dense objects come together to form less dense large "clusters" (in our terminology) with dimensions above $300 \mathrm{~nm}$ and fractal dimensions around 2. The strong interactions between fully charged lysozyme and NaPSS could be expected to 
generate aggregates (in our terminology) whose charge obstructs the formation of three-dimensional structures and so might account for the "wirelike" freeze-fracture electron micrographs observed in a recent study. ${ }^{57}$

The effect of temperature is strikingly different for the two types of coacervates. DLS spectra of PDADMAC coacervates were essentially independent of temperature, from 4 to $35^{\circ} \mathrm{C}$ (approaching protein unfolding), and rheological changes could be attributed to merely the drop in the viscosity of water, ${ }^{23}$ in contrast to the results in Figure 9, which show much greater temperature sensitivity for the chitosan coacervates. As noted above, their fluidization with increased temperature along with a dramatic decrease in relaxation time is much too large to be directly attributable to the drop in chitosan viscosity with temperature. The complexity of these temperature effects (chitosan-BSA coacervates show evidence of phase separation upon either heating to $27^{\circ} \mathrm{C}$ or cooling to $18^{\circ} \mathrm{C}$ ) suggests that temperature-dependence studies using DLS, SANS, and other methods will be needed for their elucidation.

\section{Conclusions}

We found remarkable differences with regard to both the formation and the properties for coacervates of BSA with PDADMAC versus coacervates of BSA with chitosan, two polycations of equal linear charge density but very different persistence lengths. The coacervates made with chitosan show much higher zero-shear viscosities and relaxation times, additional diffusional modes in DLS, and larger SANS scattering intensities at low $q$. To account for these differences in the framework of a reasonable interpretation of DLS, SANS, and rheology data, we propose the existence of dense and somewhat interconnected domains formed by the clustering of proteinpolyelectrolyte aggregates of variable degrees of desolvation. For PDADMAC-BSA we suggest that the size and hence connectivity of these domains is controlled by limitations of cluster growth due to aggregate charge. For chitosan-BSA, domains appear to be occupy larger coacervate volume fractions and are more interconnected but less dense. Both their reduced desolvation and larger size both arise from the lower protein charge under the conditions at which they form.

Acknowledgment. Support from the National Science Foundation (Grant No. CHE-0619039) is acknowledged. This work utilized neutron research facilities supported in part by the National Science Foundation under Agreement No. DMR0454672 and by the National Institute of Standards and Technology, U. S. Department of Commerce. We also thank the following individuals: Dr. Paul Butler, Dr. David F. R. Mildner, and Dr. Boualem Hammouda (NIST, Gaithersbug, MD) for assistance with USANS and SANS; Dr. Fabrice Cousin (Saclay) for initial neutron scattering studies of this system; Dr. Ellina Kesselman and Judith Schmidt (Technion, Israel) for CryoTEM; and Erin Sutherland and Margarita Antonov (UMassAmherst) for technical assistance.

\section{References and Notes}

(1) ISI Web of Science Home Page. http://portal.isiknowledge.com (accessed June 8, 2007).

(2) Illum, L. Pharm. Res. 1998, 15, 1326-1331.

(3) Agnihotri, S. A.; Mallikarjuna, N. N.; Aminabhavi, T. M. J. Controlled Release 2004, 100, 5-28.

(4) Janes, K. A.; Calvo, P.; Alonso, M. J. Adv. Drug Delivery Rev. 2001, 47, 83-97.

(5) Suh, J. K. F.; Matthew, H. W. T. Biomaterials 2000, 21, 25892598.
(6) Wu, L. Q.; Fernandes, R.; Yi, H.; Small, D. A.; Rubloff, G. W.; Ghodssi, R.; Bentley, W. E.; Payne, G. F. Adv. Chitin Sci. 2003, 7 , $146-149$.

(7) Rabea, E. I.; Badawy, M. E. T.; Stevens, C. V.; Smagghe, G.; Steurbaut, W. Biomacromolecules 2003, 4, 1457-1465.

(8) Itoyama, K.; Tokura, S.; Hayashi, T. Biotechnol. Prog. 1994, 10, 225-229.

(9) Magnin, D.; Dumitriu, S.; Chornet, E. J. Bioact. Compat. Polym. $\mathbf{2 0 0 3}, 18,355-373$.

(10) Kumar, M. N. V. R. React. Funct. Polym. 2000, 46, 1-27.

(11) Bailey, S. E.; Olin, T. J.; Bricka, R. M.; Adrian, D. D. Water Res. 1999, 33, 2469-2479.

(12) Takeuchi, T.; Morita, K.; Saito, T.; Kugimiya, W.; Fukamizo, T. Biosci., Biotechnol., Biochem. 2006, 70, 1786-1789.

(13) Plashchina, I. G.; Mrachkovskaya, T. A.; Danilenko, A. N.; Kozhevnikov, G. O.; Starodubrovskaya, N. Y.; Braudo, E. E.; Schwenke, K. D. In Food Colloids: Fundamentals of Formulation; Dickinson, E., Miller, R., Eds.; Special Publication 258; Royal Society of Chemistry: Cambridge, U. K., 2001; pp 293-303.

(14) Guzey, D.; McClements, D. J. Food Hydrocolloids 2006, 20, 124131.

(15) Laugel, N.; Betscha, C.; Winterhalter, M.; Voegel, J. C.; Schaaf, P.; Ball, V. J. Phys. Chem. B 2006, 110, 19443-19449.

(16) Berth, G.; Colfen, H.; Dautzenberg, H. Prog. Colloid Polym. Sci. 2002, 119, 50-57.

(17) Kong, C. Y.; Muthukumar, M. J. Chem. Phys. 1998, 109, 15221527.

(18) von Goeler, F.; Muthukumar, M. J. Chem. Phys. 1994, 100, 77967803.

(19) Manning, G. S. J. Phys. Chem. B 2003, 107, 11485-11490.

(20) Dautzenberg, H.; Goernitz, E.; Jaeger, W. Macromol. Chem. Phys. 1998, 199, 1561-1571.

(21) Trzcinski, S.; Varum, K. M.; Staszewska, D. U.; Smidsrød, O.; Bohdanecky, M. Carbohydr. Polym. 2002, 48, 171-178.

(22) Kayitmazer, A. B.; Shaw, D.; Dubin, P. L. Macromolecules 2005, 38, 5198-5204.

(23) Bohidar, H. B.; Dubin, P. L.; Majhi, P. R.; Tribet, C.; Jaeger, W. Biomacromolecules 2005, 6, 1573-1585.

(24) Kayitmazer, A. B.; Bohidar, H. B.; Mattison, K. W.; Bose, A.; Sarkar, J.; Hashidzume, A.; Russo, P. S.; Jaeger, W.; Dubin, P. L. Soft Matter 2007, 1064-1076.

(25) Schipper, N. M.; Varum, K. M.; Artursson, P. Pharm. Res. 1996, 13, 1686-1692.

(26) Anthonsen, M. W.; Varum, K.; Smidsrød, O. Carbohydr. Polym. 1993, 22, 193-201.

(27) Strand, S. P.; Tommeraas, K.; Varum, K. M.; Ostgaard, K. Biomacromolecules 2001, 2, 1310-1314.

(28) Prochazkova, S.; Varum, K. M.; Ostgaard, K. Carbohydr. Polym. 1999, 38, 115-122.

(29) Glinka, C. J.; Barker, J. G.; Hammouda, B.; Krueger, S.; Moyer, J. J.; Orts, W. J. J. Appl. Crystallogr. 1998, 31, 430-445.

(30) Kline, S. R. J. Appl. Crystallogr. 2006, 39, 895-900.

(31) Barker, J. G.; Glinka, C. J.; Moyer, J. J.; Kim, M. H.; Drews, A. R.; Agamalian, M. J. Appl. Crystallogr. 2005, 38, 1004-1011.

(32) Danino, D.; Bernheim-Groswasser, A.; Talmon, Y. Colloids Surf., A. 2001, 183-185, 113-122.

(33) Burgess, D. J. J. Colloid Interface Sci. 1990, 140, 227-238.

(34) de Kruif, C. G.; Weinbreck, F.; de Vries, R. Curr. Opin. Colloid Interface Sci. 2004, 9, 340-349.

(35) Tanford, C.; Swanson, S. A.; Shore, W. S. J. Am. Chem. Soc. 1955, 77, 6414-6421.

(36) Menon, M. K.; Zydney, A. L. Anal. Chem. 1998, 70, 1581-1584.

(37) Anthonsen, M. W.; Smidsrød, O. Carbohydr. Polym. 1995, 26, $303-$ 305.

(38) Varum, K. M.; Ottoy, M. H.; Smidsrød, O. Carbohydr. Polym. 1994, $25,65-70$.

(39) Wang, X.; Li, Y.; Lal, Y.; Huang, Q. J. Phys. Chem. B 2007, 111, 515-520.

(40) Stradner, A.; Sedgwick, H.; Cardinaux, F.; Poon, W. C. K.; Egelhaaf, S. U.; Schurtenberger, P. Nature 2004, 432, 492-495.

(41) Liu, Y.; Fratini, E.; Baglioni, P.; Chen, W. R.; Chen, S. H. Phys. Rev. Lett. 2005, 95, 118102.

(42) Zhang, F.; Skoda, M. W. A.; Jacobs, R. M. J.; Martin, R. A.; Martin, C. M.; Schreiber, F. J. Phys. Chem. B 2007, 111, 251-259.

(43) Norman, A. I.; Ivkov, R.; Forbes, J. G.; Greer, S. C. J. Chem. Phys. 2005, 123, 154904 .

(44) Beaucage, G. J. Appl. Crystallogr. 1996, 29, 134-146.

(45) Desbrieres, J. Biomacromolecules 2002, 3, 342-349. 
(46) Wang, Y.; Kimura, K.; Dubin, P. L.; Jaeger, W. Macromolecules 2000, 33, 3324-3331.

(47) Wang, Y.; Kimura, K.; Huang, Q.; Dubin, P. L.; Jaeger, W. Macromolecules 1999, 32, 7128-7134.

(48) Veis, A. J. Phys. Chem. 1961, 65, 1798-1803.

(49) Xia, J.; Dubin, P. L.; Dautzenberg, H. Langmuir 1993, 9, 20152019.

(50) Kokufuta, E.; Shimizu, H.; Nakamura, I. Macromolecules 1982, 15, $1618-1621$.

(51) Kaibara, K.; Okazaki, T.; Bohidar, H. B.; Dubin, P. L. Biomacromolecules 2000, 1, 100-107.

(52) Mattison, K. W. Ph.D. Dissertation, Purdue University, IN, 1999.
(53) Groenewold, J.; Kegel, W. K. J. Phys. Chem. B 2001, 105, $11702-$ 11709.

(54) Meyer, M.; Le Ru, E. C.; Etchegoin, P. G. J. Phys. Chem. B 2006, 110, 6040-6047.

(55) Dubin, P. L.; Murrell, J. M. Macromolecules 1988, 21, 2291-2293.

(56) Gummel, J.; Boué, F.; Deme, B.; Cousin, F. J. Phys. Chem. B 2006, 110, 24837-24846.

(57) Gummel, J.; Cousin, F.; Verbavatz, J.-M.; Boué, F. J. Phys. Chem. B 2007, 111, 8540-8546.

BM700645T 